\title{
Search for heavy resonances with the ATLAS detector
}

\section{Simone ZIMMERMANN ${ }^{* \dagger}$}

University of Bonn

E-mail: Simone.Zimmermann@eern.ch

Heavy resonances decaying into pairs of particles are an obvious signature to look for phenomena beyond the Standard Model. This article summarizes recent results on searches for resonances decaying into pairs of jets, leptons, and photons by the ATLAS collaboration at the LHC. Various models are considered such as excited quarks, neutral gauge $Z^{\prime}$ bosons, and Randall-Sundrum gravitons. Results from searches using data collected at center-of-mass energies of 7 and $8 \mathrm{TeV}$ are presented.

XXI International Workshop on Deep-Inelastic Scattering and Related Subjects -DIS2013, 22-26 April 2013

Marseilles, France

* Speaker.

${ }^{\dagger}$ on behalf of the ATLAS collaboration 


\section{Introduction}

Searches for heavy resonances in proton-proton collisions at $\sqrt{s}=8 \mathrm{TeV}$ with the ATLAS detector [1] at the LHC are presented. The analyses are performed in the invariant mass distribution of either two jets or two leptons in the event. In addition, the combination of search results in the diphoton and dilepton channels, obtained using data collected with the ATLAS detector at $\sqrt{s}=7 \mathrm{TeV}$, is reviewed.

\section{Dijet Analysis}

Resonant signals in the invariant mass distribution of the two highest $p_{\mathrm{T}}$ jets in the event are probed above the smoothly falling QCD background [2]. Possible signals could arise from excited quark models, testing the compositeness of quarks. Generic Gaussian shaped resonances of variable width are considered to constrain models predicting resonant peaks with Gaussian cores.

The integrated luminosity of the considered dataset amounts to $13.0 \mathrm{fb}^{-1}$. Events are selected by the logical OR of two central jet triggers. Each jet is required to have a transverse momentum $p_{\mathrm{T}}^{j}>150 \mathrm{GeV}$. The two highest $p_{\mathrm{T}}$ jets should be produced centrally with rapidity $|y|<2.8$ and the rapidity of the dijet center-of-mass system needs to fulfill $\left|y^{*}\right|=\left|0.5\left(y_{1}-y_{2}\right)\right|<0.6$. The invariant mass distribution of the dijet system is investigated above $1 \mathrm{TeV}$ (see Fig. 1a). The binning of the distribution is motivated by the expected resolution of the signal dijet mass estimated with the simulation. The smooth QCD background is parameterized by the four parameter function

$$
f(x)=p_{1}(1-x)^{p_{2}} x^{p_{3}+p_{4} \ln x} .
$$

The fully simulated signal template with a multiplicative constant to be determined in the fit is added to the data to account for possible influences of signal components to the background estimate. The bottom panel in Fig. 1a shows the significance of the difference between observed and

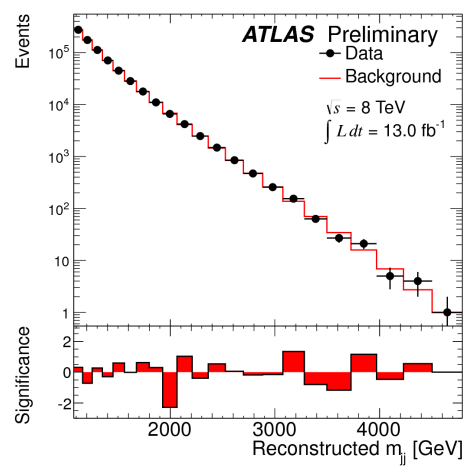

(a)

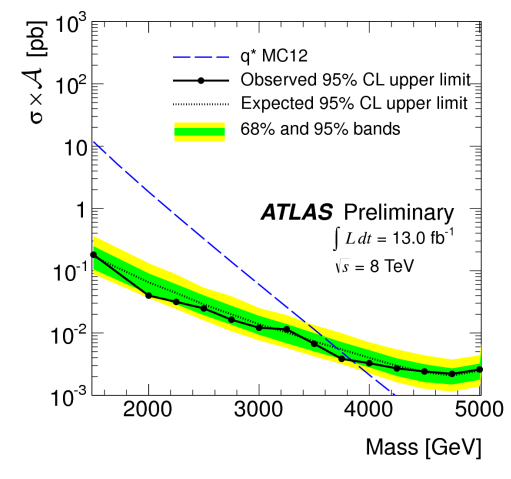

(b)

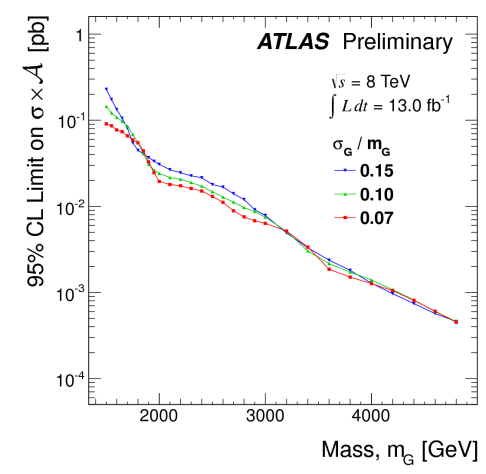

(c)

Figure 1: (a) The invariant mass distribution of the two highest $p_{\mathrm{T}}$ jets and the fit to the background hypothesis [2]. 95\% CL cross section limits from the dijet invariant mass distribution fit on (b) excited quarks and (c) generic Gaussian resonances [2]. 
predicted yield bin-by-bin in units of Gaussian standard deviations. Positive significance values correspond to data excesses and negative values to deficits. The consistency of the data with the background-only hypothesis is determined from a comparison of the $\chi^{2}$ of the fit in data with that expected from background-only pseudo-experiments, yielding a p-value of 0.61. Consequently, no significant deviation from the standard model (SM) background hypothesis is observed. Upper limits on the cross section times acceptance, $\sigma \times \mathscr{A}$, at the $95 \%$ confidence level (CL) are derived for excited quarks and generic Gaussian resonances.

In the case of excited quarks, the acceptance lies in the range of $11 \%$ to $54 \%$ depending on the excited quark mass, $m_{q^{*}}$. Figure $1 \mathrm{~b}$ compares the expected $\sigma \times \mathscr{A}$ for excited quarks as a function of $m_{q^{*}}$ with the observed 95\% CL cross section limit. From the crossing, a lower limit on the mass of a hypothetical excited quark at $m_{q^{*}}=3.84 \mathrm{TeV}$ can be set.

Three different signal widths of $\sigma_{G} / m_{G}=7 \%, 10 \%$ and $15 \%$ are considered for the modelindependent limits on Gaussian resonances. Any physics model predicting smaller widths needs to be compared to the $\sigma_{G} / m_{G}=7 \%$ results, as the dijet invariant mass resolution is on the order of $5 \%$. Figure 1c summarizes the derived $\sigma \times \mathscr{A}$ limits over the mass range explored.

\section{Dilepton Analysis}

The search for high mass dilepton resonances [3] is based on an integrated luminosity of $20.0 \mathrm{fb}^{-1}$. Two distinct models predicting a new neutral gauge boson $Z^{\prime}$, the Sequential Standard Model (SSM) and Grand Unification Models with an additional $E_{6}$ gauge group, are considered. In the SSM benchmark model the $Z^{\prime}$ has the same couplings to leptons as the SM $Z$ boson and a width of $\sigma_{Z_{\text {SSM }}^{\prime}} / m_{Z_{S S M}^{\prime}}=3.1 \%$. The lightest linear combination of two new neutral gauge bosons from the $E_{6}$ gauge group

$$
Z^{\prime}\left(\theta_{6}\right)=Z_{\Psi}^{\prime} \cos \theta_{6}+Z_{\chi}^{\prime} \sin \theta_{6}
$$

is determined by the mixing angle $\theta_{6}$ and the pattern of the electroweak symmetry breaking. In total, six different models are investigated with widths in the range of $\sigma_{Z^{\prime}\left(\theta_{E_{6}}\right)} / m_{Z^{\prime}\left(\theta_{E_{6}}\right)}=0.5 \%$ to $1.3 \%$. Moreover, excited Kaluza-Klein modes of the graviton predicted by the Randall-Sundrum model of extra spatial dimensions are tested. Model parameters are the space-time curvature of the extra dimension, $k$, and the reduced Planck mass, $\bar{M}_{\mathrm{Pl}}=M_{\mathrm{Pl}} / \sqrt{8 \pi}$. The first excitation $G^{*}$ of these modes appears as a spin-2 narrow-width resonance (e.g. $\sigma_{G^{*}} / m_{G^{*}}=1.4 \%$ for $k / \bar{M}_{\mathrm{Pl}}=0.1$ ).

The search is performed in two channels, the decay to $e^{+} e^{-}$and to $\mu^{+} \mu^{-}$. The selection in the electron channel is based on a diphoton trigger of $E_{\mathrm{T}}>35 \mathrm{GeV}$ and $E_{\mathrm{T}}>25 \mathrm{GeV}$, which has advantages over dielectron triggers for the background estimation. Events are selected that contain at least two isolated electron candidates within $|\eta|<2.47$ with $E_{\mathrm{T}}>40 \mathrm{GeV}$ and $E_{\mathrm{T}}>30 \mathrm{GeV}$. The resulting dielectron invariant mass distribution for the electron pair with the highest $\sum p_{\mathrm{T}}$ is shown in Fig. 2a. At $m_{e e}=2 \mathrm{TeV}$ the acceptance times efficiency is $73 \%$. Similarily, the muon selection relies on the OR of two single muon triggers of $p_{\mathrm{T}}=24 \mathrm{GeV}$ and $p_{\mathrm{T}}=36 \mathrm{GeV}$ thresholds. Each muon is required to have $p_{\mathrm{T}}>25 \mathrm{GeV}$ and $|\eta|<2.4$. The resulting dimuon invariant mass distribution for the muon pair with the highest $\sum p_{\mathrm{T}}$ is shown in Fig. 2b. The acceptance times efficiency at $m_{\mu \mu}=2 \mathrm{TeV}$ amounts to $46 \%$.

Data-driven estimates of the $W+$ jets and QCD multijet backgrounds are combined with Monte Carlo predictions of the $t \bar{t}$, diboson and the irreducible $Z / \gamma^{*} \rightarrow l^{+} l^{-}$backgrounds. The sum of the 


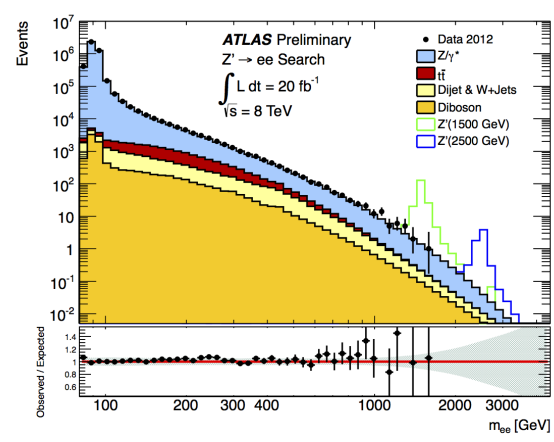

(a)

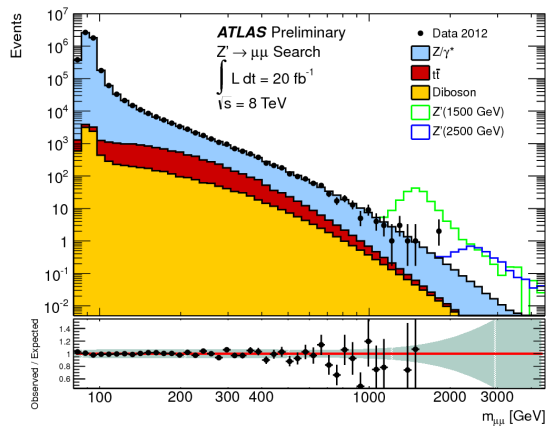

(b)

Figure 2: The dilepton invariant mass distribution in the (a) $e^{+} e^{-}$and (b) $\mu^{+} \mu^{-}$channels. Data (points with error bars) are compared to the SM background prediction, as well as to the expected distributions from hypothetical Z' bosons with mass of $1500 \mathrm{GeV}$ and $2000 \mathrm{GeV}$, respectively [3].

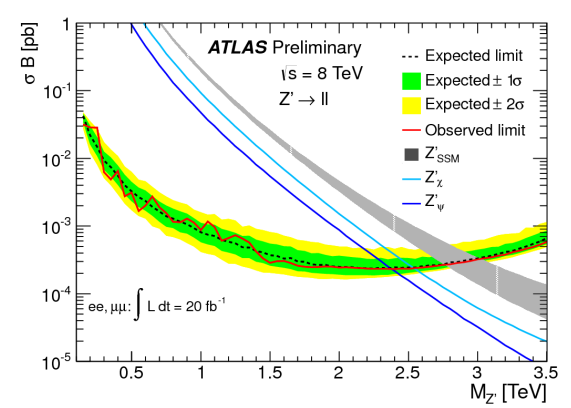

(a)

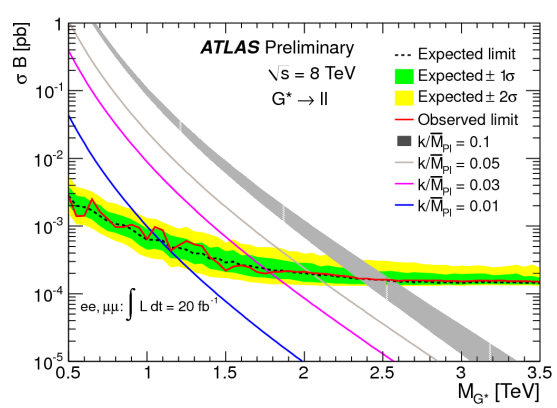

(b)

Figure 3: Expected and observed 95\% CL upper limits on cross section times branching ratio for (a) a Z' boson and (b) a Randall-Sundrum graviton decaying into dileptons as a function of resonance mass, resulting from the combination of the $e^{+} e^{-}$and $\mu^{+} \mu^{-}$channels. Also shown are the theoretical predictions for different benchmark models [3].

Monte Carlo estimates, where the relative contributions are fixed by their predicted cross sections, is scaled to agree with the data yield in the normalization region around the $Z$ boson peak: $80 \mathrm{GeV}<m_{l l}<110 \mathrm{GeV}$. Figure 2 shows the invariant mass distributions in the two search channels as measured in data and the estimated SM background contributions.

The 95\% CL upper cross section limits are derived separately in the electron and muon channel and combined assuming equal branching fractions. They are interpreted in Fig. 3a with respect to the SSM, resulting in a lower mass limit of $2.86 \mathrm{TeV}$ for the $Z_{\mathrm{SSM}}^{\prime}$ boson, and with respect to $E_{6^{-}}$ motivated models yielding lower mass limits in the range of $2.38-2.54 \mathrm{TeV}$ (see Table 1). The derived cross section limits on the Randall-Sundrum graviton $G^{*}$ are shown in Fig. 3b. Assuming $k / \bar{M}_{\mathrm{Pl}}=0.1$, the resulting lower mass limit is $m_{G^{*}}>2.47 \mathrm{TeV}$. 
Table 1: The observed 95\% CL lower mass limits on six different $E_{6}$ gauge group $Z^{\prime}$ models [3].

\begin{tabular}{lcccccc}
\hline model & $Z_{\Psi}^{\prime}$ & $Z_{\eta}^{\prime}$ & $Z_{N}^{\prime}$ & $Z_{I}^{\prime}$ & $Z_{S}^{\prime}$ & $Z_{\chi}^{\prime}$ \\
limit & $2.38 \mathrm{TeV}$ & $2.39 \mathrm{TeV}$ & $2.44 \mathrm{TeV}$ & $2.42 \mathrm{TeV}$ & $2.47 \mathrm{TeV}$ & $2.54 \mathrm{TeV}$ \\
\hline
\end{tabular}

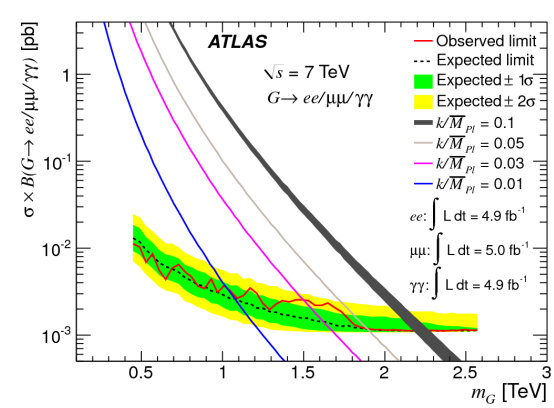

(a)

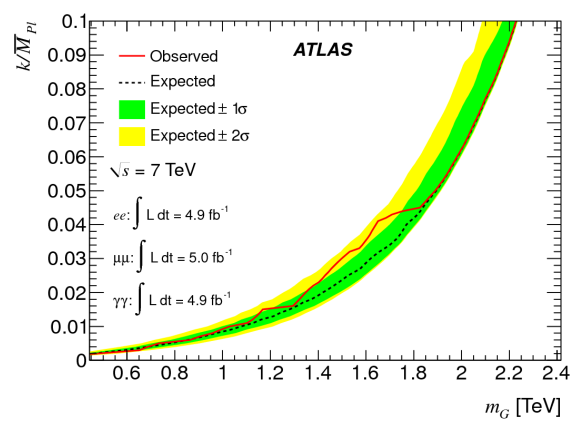

(b)

Figure 4: (a) Expected and observed 95\% CL upper limits on cross section times branching ratio for a Randall-Sundrum graviton as a function of $m_{G^{*}}$, resulting from the combination of searches in the $e^{+} e^{-}, \mu^{+} \mu^{-}$and $\gamma \gamma$ decay modes. Also shown are the theoretical predictions for different values of $\mathrm{k} / \mathrm{Mpl}$ [5]. (a) Excluded region in the plane of $m_{G^{*}}$ and $k / \bar{M}_{\mathrm{Pl}}$ [5].

\section{Combination of Dilepton and Diphoton Analysis Results}

Randall-Sundrum gravitons decaying into dileptons [4] an diphotons [5] have been searched for using the complete 2011 data set collected with ATLAS at $\sqrt{s}=7 \mathrm{TeV}$. The combined cross section limits, assuming equal branching fractions to electrons and muons and a two times higher branching fraction to photons, is shown as a function of $m_{G^{*}}$ in Fig. 4a. The combination excludes $G^{*}$ below $m_{G^{*}}=2.23 \mathrm{TeV}(1.00 \mathrm{TeV})$ for $k / \bar{M}_{\mathrm{Pl}}=0.1(0.01)$, improving the dilepton sensitivity by $\sim 100 \mathrm{GeV}$. The excluded area in the plane of $m_{G^{*}}$ and $k / \bar{M}_{\mathrm{Pl}}$ is illustrated in Fig. $4 \mathrm{~b}$.

\section{Summary}

We have presented the results of searches for heavy resonances decaying into dijets, dileptons and diphotons using $p p$ collision data corresponding to $5 \mathrm{fb}^{-1}$ at $\sqrt{s}=7 \mathrm{TeV}$ and up to $20 \mathrm{fb}^{-1}$ at $\sqrt{s}=8 \mathrm{TeV}$ collected with the ATLAS detector. No deviations from SM predictions are observed and cross section limits and mass exclusions are derived for several extensions of the SM.

\section{References}

[1] ATLAS Collaboration, JINST 3 S08003 (2008).

[2] ATLAS Collaboration, ATLAS-CONF-2012-148, https://cds.cern.ch/record/1493487.

[3] ATLAS Collaboration, ATLAS-CONF-2013-017, https://cds.cern.ch/record/1525524.

[4] ATLAS Collaboration, Phys. Rev. D 87, 015010 (2013).

[5] ATLAS Collaboration, New J. Phys. 15 (2013) 043007. 